\title{
ODD ORDER GROUPS WITH AN AUTOMORPHISM CUBING MANY ELEMENTS
}

\author{
MARIAN DEACONESCU and DESMOND MacHALE
}

(Received 3 July 1987; revised 27 January 1988)

Communicated by H. Lausch

\begin{abstract}
We determine the structure of a nonabelian group $G$ of odd order such that some automorphism of $G$ sends exactly $(1 / p)|G|$ elements to their cubes, where $p$ is the smallest prime dividing $|G|$. These groups are close to being abelian in the sense that they either have nilpotency class 2 or have an abelian subgroup of index $p$.
\end{abstract}

1980 Mathematics subject classification (Amer. Math. Soc.) (1985 Revision): 20 E 36.

\section{Introduction}

Let $G$ be a group and let $n$ be a fixed non-zero integer. An $n$-automorphism of $G$ is an automorphism which sends every element of $G$ to its $n$th power. If $G$ has an $n$-automorphism for $n=-1,2$ or 3 , it is well known that $G$ is abelian. On the other hand, Miller [8] has shown that for every other value of $n \neq 1$ there exists a non-abelian group admitting a non-trivial $n$-automorphism.

For a finite non-abelian group $G$ and for $n=-1,2$ and 3 , there remains the problem of determining how large a proportion of the elements of $G$ can be sent to their $n$th powers by an automorphism, and also of determining the structure of the groups for which these maximal proportions are achieved. For $n=-1$ and 2, these problems were solved by Manning [7], Liebeck and MacHale [2], and Liebeck [4]. (See also MacHale [5], and [3].) Concerning $n=3$, the following results are proved in MacHale [6].

(C) 1989 Australian Mathematical Society $0263-6115 / 89 \$ A 2.00+0.00$ 
(i) No automorphism of $G$ can send more than $(3 / 4)|G|$ elements to their cubes.

(ii) $G$ has an automorphism cubing exactly $(3 / 4)|G|$ elements if and only if $|G: Z(G)|=4$ and $Z(G)$ has no elements of order 3 , where $Z(G)$ is the centre of $G$.

(iii) If $|G|$ is odd and $p$ is the least prime dividing $|G|$, then no automorphism of $G$ can send more than $(1 / p)|G|$ elements to their cubes.

In this paper we settle the outstanding case arising from (iii) above by classifying all non-abelian groups $G$ of odd order with an automorphism $\alpha$ which sends exactly $(1 / p)|G|$ elements to their cubes, where $p$ is the least prime dividing $|G|$.

Let $\left.T=T_{\alpha}=\left\{x \in G \mid x \alpha=x^{3}\right)\right\}$ and $F=F_{\alpha}=\{x \in G \mid x \alpha=x\}$. The classification theorem depends on whether $F$ is trivial or not.

THEOREM. (i) If $|F|=1$, then $G$ is nilpotent of class $2,\left|G^{\prime}\right|=p, G^{p} \cap G^{\prime}=1$ and $p \geq 5$.

(ii) If $|F| \neq 1$, then $T$ is an abelian subgroup of index $p$ in $G$ and there exists $f \in F, f \notin T$, such that $f$ has order $p$. Moreover $(|T|, 3)=1$.

These conditions are necessary and sufficient. Thus, as in the cases $n=-1$ and 2 , the groups in question are close to being abelian in that they either have small nilpotency class or an abelian subgroup of small index.

\section{Notation}

Throughout, $G$ will denote a finite group of odd order. Any notation not explicitly defined is standard and conforms to that of [1].

$\mathscr{G}_{p}$ the set of all finite groups with order divisible by the prime $p$ but by no smaller prime.

$\alpha$ an automorphism of $G$,

$T_{\alpha}=T$, the set $\left\{x \in G \mid x \alpha=x^{3}\right\}$,

$F_{\alpha}=F$, the subgroup $\{x \in G \mid x \alpha=x\}$,

$G^{p}$ the subgroup generated by the $p$ th powers of elements of $G$,

$|x|$ the order of the element $x \in G$,

$x^{G}$ the conjugacy class of $G$ containing $x$,

$Z(G)=Z$, the centre of $G$.

\section{Preliminary results}

The following remarks are at once obvious. 
(i) $T \cap F=1$, since $|G|$ is odd.

(ii) $(T) \alpha=T$.

(iii) No element of $T$ has order divisible by 3 .

(iv) If $A$ is a subgroup of $G$, maximal in $T$, then $(A) \alpha=A$ and $A$ is abelian, since the resctriction of $\alpha$ to $A$ is a 3-automorphism of $A$.

LEMMA 3.1. If $G \in \mathscr{G}_{p}$ and $H \triangleleft G$ with $|H|=p$, then $H \subset Z(G)$.

Proof. Let $H=\langle h\rangle$. Now all the conjugates of $h$ lie in $H$ and their number, being a divisor of $|G|$, is either 1 or $p$. Since the identity is not conjugate to $h, h$ has exactly one conjugate. Thus $h$ is central and the result follows.

LEMMA 3.2. If $t \in T, C_{G}(t)=C_{G}\left(t^{3}\right)$.

ProOF. If $t g=g t$ then $t^{3} g=g t^{3}$. Conversely, if $t^{3} g=g t^{3}$ then applying the automorphism $\alpha^{-1}, t\left(g \alpha^{-1}\right)=\left(g \alpha^{-1}\right) t$. Since the correspondence $g \leftrightarrow g\left(\alpha^{-1}\right)$ is one-to-one, the result follows.

LEMMA 3.3. If $\alpha$ is fixed-point-free, then any conjugacy class of $G$ contains at most one element of $T$.

ProOF. For $g \in G, t \in T$, suppose that $g^{-1} t g \in T$. Then $\left(g^{-1} t g\right) \alpha=$ $\left(g^{-1} t g\right)^{3}$, whence $\left[g(g \alpha)^{-1}, t^{3}\right]=1$. By Lemma $3.2,\left[g(g \alpha)^{-1}, t\right]=1$, and since $\alpha$ is fixed-point-free, this implies $[g, t]=1$, as claimed.

LEMMA 3.4. If $G \in \mathscr{G}_{p}$ has $k$ conjugacy classes, then

$$
\frac{k}{|G|} \leq \frac{1}{p^{2}}\left[1+\frac{p^{2}-1}{\left|G^{\prime}\right|}\right] \text {. }
$$

Proof. $G$ has $\left|G: G^{\prime}\right|$ irreducible representations of degree 1 and all other ones have degrees at least $p$. The degree equation, $|G|=\sum_{i=1}^{k} d_{i}^{2}$, now gives $|G| \geq\left|G: G^{\prime}\right|+\left(k-\left|G: G^{\prime}\right|\right) p^{2}$, from which the result follows.

LEMMA 3.5. If $G \in \mathscr{G}_{p}$ and $|F|>1$, then $T f_{1}=T f_{2}$ implies $f_{1}=f_{2}$, for $f_{1}, f_{2} \in F$. In this case $G=T F=F T$ and $|F|=p$.

PROOF. Suppose $f_{1}=t f_{2}$, for $t \in T$. Applying $\alpha$, we have $f_{1}=t^{3} f_{2}=t^{2} f_{1}$. Since $|G|$ is odd, $t=1$ and $f_{1}=f_{2}$. Now any $f \in F, f \neq 1$, has order at least $p$ so $G=T \cup T f \cup \cdots \cup T f^{p-1}$. Thus $G=T F$ and $|F|=p$. Similarly, $G=F T$. We assume from now on that $G$ is a non-abelian group in $\mathscr{G}_{p}(p>2)$ and some automorphism $\alpha$ of $G$ satisfies $p\left|T_{\alpha}\right|=p|T|=|G|$. 


\section{Case where $|F|=1$}

We assume throughout this section that $\alpha$ is fixed-point-free. In this case we claim that $T$ cannot be a subgroup, so suppose otherwise. Then $|G: T|=p$, $T \triangleleft G$ and so $T$ consists of complete conjugacy classes in $G$. Then, by Lemma $3.3, T \subset Z(G), G / Z(G)$ is cyclic and $G$ is abelian, a contradiction.

Suppose that $G$ has $k$ conjugacy classes. Then, by Lemma $3.3, k \geq|T|$, so $k /|G| \geq|T| /|G|=1 / p$. By Lemma 3.4

$$
\frac{1}{p^{2}}\left[1+\frac{p^{2}-1}{\left|G^{\prime}\right|}\right] \geq \frac{1}{p}
$$

whence $\left|G^{\prime}\right| \leq p+1$. Since $G \in \mathscr{G}_{p}$ and $p$ is odd, $\left|G^{\prime}\right|=p$. By Lemma 3.1, $G^{\prime} \subset Z(G)$, so $G$ is nilpotent of class 2 .

Next, we show $p \neq 3$, so suppose $p=3$. Let $a, b \in T$ be such that $[a, b] \neq 1$. Such a pair of elements exists since otherwise $T$ is a subgroup, a contradiction. Since $G^{\prime}$ is characteristic in $G$ and $\alpha$ is fixed-point-free, $[a, b]^{2}=[a, b] \alpha=$ $\left[a^{3}, b^{3}\right]=[a, b]^{9}$. Thus $[a, b]^{7}=1$ and so $[a, b]=1$, a contradiction. Thus $p \geq 5$.

We now claim that $Z=Z(G) \not \subset T$, so suppose otherwise. Since then $G^{\prime} \subset$ $Z \subset T, G^{\prime} \subset T$. If $a, b \in T,[a, b] \neq 1$ then

$$
[a, b] \alpha=[a, b]^{3}=\left[a^{3}, b^{3}\right]=[a, b]^{9} .
$$

Thus $[a, b]^{6}=1$, which forces $[a, b]=1$, since $|G|$ is odd and $T$ has no elements of order 3. This contradiction shows $Z \not \subset T$.

Let $Z^{*}=Z \cap T$. Then $Z^{*}$ is a subgroup of $Z$ with $\left|Z: Z^{*}\right|=p$. To see this, consider $Z t \cap T$ for any $t \in T$. Let $z \in Z$. Now $z t \in T \Leftrightarrow(z t)^{3}=z \alpha t^{3} \Leftrightarrow$ $z \alpha=z^{3} \Leftrightarrow z \in Z^{*}$. Thus $Z t \cap T=Z^{*} t$. If $\left|Z: Z^{*}\right|>p$, then $|T|<(1 / p)|G|$, a contradiction. For $a, b \in T$, if $1 \neq[a, b]=c$ then $c$ generates $G^{\prime}$, and $c \notin Z^{*}$, since then $c^{3}=c \alpha=\left[a^{3}, b^{3}\right]=c^{9}$ and $c=1$. Thus, $Z=Z^{*} \times G^{\prime}$.

Finally, we show that $G^{p} \cap G^{\prime}=1$. Now, since $G$ has class 2 , for all $t \in T$, $x \in G,\left[t^{p}, x\right]=[t, x]^{p}=1$, so $t^{p} \in Z \cap T=Z^{*}$. Thus, for all $a, b \in T$, $(a b)^{p}=a^{p} b^{p}[b, a]^{p(p-1) / 2}=a^{p} b^{p}$, since $p$ is odd. Thus $G^{p} \subset Z^{*}$, so $G^{p} \cap G^{\prime}=1$.

We can now state a structure theorem in the case $F=1$.

THEOREM 4.1. Necessary and sufficient conditions that a non-abelian group $G \in \mathscr{G}_{p}(p>2)$ have an automorphism $\alpha$ such that $\left|F_{\alpha}\right|=1$ and $p\left|T_{\alpha}\right|=G$ are

(i) $G$ is nilpotent of class 2 with $\left|G^{\prime}\right|=p$,

(ii) $G^{p} \cap G^{\prime}=1$ and

(iii) $p \geq 5$.

ProOF. We have already established the necessity of these conditions. Suppose that $G$ is a group which satisfies (i)-(iii). Then $G / Z$ is an elementary 
abelian p-group and $Z=Z^{*} \times G^{\prime}$, where $G^{p} \subset Z^{*} \subset G$. Thus $G / Z=$ $\left\langle Z a_{1}, \ldots, Z a_{k}, Z x_{1}, \ldots, Z x_{k}\right\rangle$, where $\left[x_{i}, x_{j}\right]=\left[a_{i}, a_{j}\right]=1$ for all $i, j=1, \ldots, k$; $\left[a_{i}, x_{j}\right]=1(i \neq j) ;\left[a_{i}, x_{i}\right]=c(i=1, \ldots, k)$, where $\langle c\rangle=G^{\prime}$. Put $A=$ $\left\langle a_{1}, \ldots, a_{k}, Z^{*}\right\rangle$. Then every element $g \in G$ is uniquely expressible as $g=$ $a c^{s} x_{1}^{q_{1}} \cdots x_{k}^{q_{k}}$, where $a \in A, 0 \leq s \leq p-1,0 \leq q_{i}<p(i=1, \ldots, k)$. The map $\alpha$ defined by

$$
g \alpha=\left(a c^{s} x_{1}^{q_{1}} \cdots x_{k}^{q_{k}}\right) \alpha=a^{3} c^{9 s} x_{1}^{3 q_{1}} \cdots x_{k}^{3 q_{k}}
$$

defines an automorphism of $G$. Moreover, $p\left|T_{\alpha}\right|=G$ because, given any $a \in A$ and integers $q_{1}, \ldots, q_{k}$, there is exactly one $s, 0 \leq s<p$, such that $g \alpha=g^{3}$. Finally, we note that $\left|G_{\alpha}\right|=1$ since $p \geq 5$.

\section{Groups in which $p|T|=|G|$ and $|F| \neq 1$}

The analysis in this section resembles section $4 B$ of Liebeck [4]. However, it differs in detail and the outcome is different.

Up to the end of this section we shall assume the following conditions: $G \in$ $\mathscr{G}_{p}(p>2)$ is a non-abelian group, $\alpha \in \operatorname{Aut}(G)$ with $p|T|=p\left|T_{\alpha}\right|=|G|$ and $F=F_{\alpha} \neq 1$.

By Lemma $3.5,|F|=p$, so if $F=\langle f\rangle$ we have the disjoint union

$$
G=T \cup T f \cup \cdots \cup T f^{p-1}=T \cup f T \cup \cdots \cup f^{p-1} T .
$$

LEMMA 5.2. The conjugacy class containing $f$ has no elements in $T$.

Proof. Suppose there exists $g \in G$ such that $g^{-1} f g \in T$. By (0), $g=t f^{r}$, for $t \in T$ and some integer $r$. Thus $\left(f^{-r} t^{-1} f t f^{r}\right) \alpha=\left(f^{-r} t^{-1} f t f^{r}\right)^{3}$, whence $t^{-2} f t^{2}=f^{3}$. Applying $\alpha$, we have $t^{-6} f t^{6}=f^{3}$, from which $f=t^{-4} f t^{4}$, and so $t^{-1} f t=f$, since $|G|$ is odd. Finally, $t^{-2} f t^{2}=f=f^{3}$, so $f=1$, a contradiction.

LEMMA 5.3. The conjugacy class of $t \in T$ either has one element in $T$ when $[t, f]=1$, or has exactly $p$ elements in $T$, when $[t, f] \neq 1$. These elements are $f^{-r} t f^{r}, r=0,1, \ldots, p-1$.

Proof. Let $g \in G$ and $t \in T$ with $g^{-1} t g \in T$. Then from $\left(g^{-1} t g\right) \alpha=$ $\left(g^{-1} t g\right)^{3}$ we find that $\left[(g \alpha) g^{-1}, t^{3}\right]=1$, implies $\left[(g \alpha) g^{-1}, t\right]=1$. But $g=t_{1} f^{r}$ for some $t_{1} \in T$ and some integer $r$, so $\left(t_{1} f^{r}\right) \alpha\left(t_{1} f^{r}\right)^{-1} t=t\left(t_{1} f^{r}\right) \alpha\left(t_{1} f^{r}\right)^{-1}$. This simplifies to $t_{1}^{2} t=t t_{1}^{2}$, so $t_{1} t=t t_{1}$ and $g^{-1} t g=f^{-r} t f^{r}$, which proves the assertion of the lemma. 
LEMMA 5.4. Suppose that $T$ is not a subgroup of $G$. If $x, y$ and $x y$ all belong to $T$, then $x y=y x$.

ProOF. Suppose $x, y$ and $x y$ belong to $T$. Then since $|x y|=|y x|$, we have $3 \nmid|x||y||x y||y x|$. Applying $\alpha$, we obtain $(x y)^{3}=x^{3} y^{3}$, so

$$
(y x)^{2}=x^{2} y^{2}
$$

By (0), yx $=f t$, for some $f \in F, t \in T$, and applying $\alpha$, we get $y^{3} x^{3}=f t^{3}=$ ft. $t^{2}=y x t^{2}$. Thus $x^{-1} y^{2} x^{3}=t^{2}=x^{-1}\left(y^{2} x^{2}\right) x$. Now conjugating (1) by $y^{-2}$ gives $\left(y^{3} x y^{-2}\right)^{2}=y^{2} x^{2}$ and substituting gives $t=x^{-1} y^{3} x y^{-2} x$, since $t^{2}=u^{2}$ implies $t=u$. Hence $y x=f t=f x^{-1} y^{3} x y^{-2} x$, so

$$
y^{3}=f x^{-1} y^{2} x \text {. }
$$

Applying $\alpha$ to (2) we get

$$
y^{9}=f x^{-3} y^{9} x^{3} .
$$

Combining (2) and (3) yields $y^{6}=x^{-1} y^{-3} x^{-2} y^{9} x^{3}=(y x)^{-1}\left(y^{-2} x^{-2}\right) y^{9} x^{3}=$ $(y x)^{-3} y^{9} x^{6}$ from (1). Thus $(y x)^{3}=y^{9} x^{3} y^{-6}=y^{9} x^{3} y^{3} y^{-9}=\left[y^{9}(x y) y^{-9}\right]^{3}$. Since $3 \nmid|x y||y x|$, we conclude that $y x=y^{9} x y y^{-9}$ so $x y^{8}=y^{8} x$ and $x y=y x$.

LEMMA 5.5. Suppose that $T$ is not a subgroup of $G$. Let $A$ be a subgroup of $G$ maximal in $T$. Then there exists a coset decomposition

$$
G=A \cup A f \cup \cdots \cup A f^{p-1} \cup A g_{1} \cup \cdots \cup A g_{n}
$$

such that

(i) $A f^{j} \cap T=\phi, j=1,2, \ldots, p-1$, and

(ii) $\left|A g_{i} \cap T\right|=\left|C_{A}\left(g_{i}\right)\right|=|A| / p, i=1,2, \ldots, n$.

PrOOF. (i) is a consequence of (0).

(ii) Clearly, exactly $1 / p$ of the elements of $A \cup A f \cup \cdots \cup A f^{p-1}$ belong to $T$. For $t \in T \backslash A$ we have $A t \cap T=C_{A}(t) t$ by Lemma 5.4. Since $A$ is abelian and maximal in $T, C_{A}(A t)=C_{A}(t)$ is a proper subgroup of $A$. Consequently $|A g \cap T| \leq|A| / p$ for all $g \in G \backslash A$. It follows that every coset $A g_{i}$ must have exactly $1 / p$ of its elements in $T$, otherwise the condition $p|T|=|G|$ is violated. Hence $\left|A g_{i} \cap T\right|=|A| / p$ for $i=1,2, \ldots, n$.

We now proceed to prove the following result, which, together with the corollary below and Theorem 4.1 establishes the characterisation theorem stated at the outset. 
THEOREM 5.6. If $G \in \mathscr{G}_{p}(p>2)$ is non-abelian and has an automorphism $\alpha$ such that $F_{\alpha} \neq 1$ and $p|T|=|G|$, then $T$ is a subgroup of $G$.

PROOF. We proceed by induction on $|G|$. Assume first that $Z^{*}=Z(G) \cap T \neq$ 1. It is clear that $Z^{*}$ is an $\alpha$-invariant normal subgroup of $G$. If $G^{\prime} \subset Z^{*}$, then for all $a, b \in T$ by "bilinearity"

$$
[a, b]^{3}=[a, b] \alpha=[a \alpha, b \alpha]=\left[a^{3}, b^{3}\right]=[a, b]^{9} .
$$

This implies that $[a, b]=1$ as $|G|$ is odd and $T$ has no element of order 3 . We may infer that $T$ is a subgroup of $G$.

If $G / Z^{*}=\left(F Z^{*} / Z^{*}\right)\left(T / Z^{*}\right)$ is non-abelian, it satisfies all hypotheses of the theorem, in view of the statement (iii) in the introduction and Lemma 3.5. Thus by induction $T / Z^{*}$ and hence $T$ are groups. We may therefore assume that $Z^{*}=1$.

We claim that there is a $g \in G$ such that $\left|g^{G} \cap T\right|=p$ and $\left|g^{G}\right|<p^{2}$. Assume the contrary. If $\left|x^{G} \cap T\right| \neq p$ for some $1 \neq x \in G$, then either $x^{G} \cap T=\varnothing$ or $\left|x^{G} \cap T\right|=1$ and $\left|x^{G}\right|=\left|G: C_{G}(x)\right| \geq p$, by Lemma 5.3. We know that $Z^{*}=1$ and $G \in \mathscr{G}_{p}$, so from $|G|=p|T|$ (and our assumption) we may conclude that the union of all conjugacy classes of $G$ intersecting $T$ trivially contains at most $p-1$ elements. Combining Lemmas 5.2 and 3.5 we obtain that $F=Z(G)$ is this union with 1 added.

Since $\alpha$ induces on $G / F$ a 3 -automorphism by (0), we get $G^{\prime} \subseteq F$. Now for all $a, b \in T,[a, b]=[a, b] \alpha=\left[a^{3}, b^{3}\right]=[a, b]^{9}$, implying that $[a, b]=1$. It follows that $G$ is abelian, a contradiction.

Hence there is a $g \in G$ such that $\left|g^{G} \cap T\right|=p$ and $\left|g^{G}\right|<p^{2}$. By Lemma 5.3, $A=C_{G}(g)$ does not contain $F$, whence $A \cap F=1$. In view of Lemma 3.2, $A$ is $\alpha$-invariant. For any $a \in A$ there exist $j$ such that $a f^{j} \in T$ by $(0)$, so $\left(a f^{j}\right)^{3}=\left(a f^{j}\right) \alpha=a^{\alpha} f^{j}$ implies $\left(f^{j} a\right)^{2}=a^{-1} a \alpha \in A$. It follows that $f^{j} a \in A$ and $f^{j} \in A \cap F=1$, whence $j=0$ and $a \in T$. Thus $A \subset T$, so $A$ is abelian. We claim that $A=T$.

Assuming the contrary we have $p<|G: A|<p^{2}$. Since $G \in \mathscr{G}_{p},|G: A|=$ $\left|g^{G}\right|$ must be a prime $q$, say. In particular, $A$ is a maximal subgroup of $G$. There exists $t \in T \backslash A$. By Lemma 5.5, $\left|C_{A}(t)\right|=|A| / p$. On the other hand, $C_{G}\left(C_{A}(t)\right) \supseteq\langle A, t\rangle=G$. Since $Z^{*}=1$, we obtain $C_{A}(t)=1,|A|=p$ and $|G|=p q$. But now $A$ and $F$ are conjugate in $G$ (Sylow), contradicting Lemma 5.2. The proof is complete.

From the proof of Theorem 5.6 we have

COROLLARY 5.7. A non-abelian group $G \in \mathscr{G}_{p}(p>2)$ has an automorphism $\alpha$ such that $F \neq 1$ and $T$ is a subgroup of index $p$ in $G$ if and only if $G$ has 
an abelian subgroup $A$ of index $p$ with $(|A|, 3)=1$ and an element $f \in G \backslash A$ of order $p$.

\section{Acknowledgement}

We wish to thank Professor T. J. Laffey for his valuable help in the construction of this paper. Thanks are due also to the referee, whose useful remarks led to a shortened version of the proof of Theorem 5.6.

\section{References}

[1] D. Gorenstein, Finite groups (Harper and Row, New York, 1968).

[2] H. Liebeck and D. MacHale, 'Groups with automorphisms inverting most elements', Math. Z. 124 (1972), 51-63.

[3] H. Liebeck and D. MacHale, 'Groups of odd order with automorphisms inverting many elements', J. London Math. Soc. 6 (1973), 215-225.

[4] H. Liebeck, 'Groups with an automorphism squaring many elements', J. Austral. Math. Soc. 16 (1973), 33-42.

[5] D. MacHale, 'Universal power automorphisms in finite groups', J. London Math. Soc. 2 (1975), 366-368.

[6] D. MacHale 'Groups with an automorphism cubing many elements', J. Austral. Math. Soc. 2 (1975), 253-256.

[7] W. A. Manning, 'Groups in which a large number of operators may correspond to their inverses', Trans. Amer. Math. Soc. 7 (1906), 233-240.

[8] G. A. Miller, 'Possible $\alpha$-automorphisms of non-Abelian groups', Proc. Nat. Acad. Sci. U.S.A. 15 (1929), 89-91.

Department of Mathematics

University of Timisoara

1900-Timisoara

Romania
Department of Mathematics

University College

Cork

Ireland 